\title{
A Method of Reducing Systematic Errors in Classification Problems
}

\author{
Louis Lyons
}

PPE Division, CERN, Geneva, Switzerland

\begin{abstract}
In classification problems, the usual way of estimating systematic errors consists of first deciding on an algorithm for determining the quantity of interest, and then seeing the effect on the answer of varying the relevant (Monte Carlo) input constants by suitable amounts. A better way of analysing the date, which can result in reduced systematic errors, is described. Its effect on a toy example is presented; the improvement is striking.
\end{abstract}

*) Permanent Address : Nuclear Physics Laboratory, Keble Road, Oxford, UK. 

CERN-PPE/92

21 July 1992

\title{
A METHOD OF REDUCING SYSTEMATIC ERRORS IN CLASSIFICATION PROBLEMS
}

\author{
Louis Lyons* \\ PPE Division \\ CERN, Geneva, Switzerland
}

\begin{abstract}
In classification problems, the usual way of estimating systematic errors consists of first deciding on an algorithm for determining the quantity of interest, and then seeing the effect on the answer of varying the relevant (Monte Carlo) input constants by suitable amounts. A better way of analysing the data, which can result in reduced systematic errors, is described. Its effect on a toy example is presented; the improvement is striking.
\end{abstract}

*) Permanent address: Nuclear Physics Laboratory, Keble Road, Oxford, UK. 



\section{Introduction}

In High Energy Physics, and in many other situations too, we are often interested in dividing observations into different categories. Thus we may wish to determine what fraction of interactions between electrons and positrons into hadronic final states are mediated by beauty quarks or by other lighter ones; or which of a group of men are Japanese. Such separation methods require some algorithm which enables us to determine the fraction of interest. For example, perhaps our discrimination between Japanese and other men is based on their height. To devise such an algorithm, we would need information on the expected height distributions for the two categories.

In such determinations (as in any measurement), it is necessary to estimate not only the statistical error arising from the limited sample size, etc., but also any possible systematic error. The latter could arise from our assumed distributions for the heights of Japanese and other men being incorrect or irrelevant (maybe these men are all athletes, whose heights are not typical of the general population).

The conventional method for estimating possible systematic effects is to vary the relevant parameters of the expected distributions by some reasonable amount, and to see the extent to which this affects the answer from the previously defined algorithm for estimating the fraction. Thus we may vary the mean height of the expected distribution for Japanese men; or the width of the corresponding distribution for 'others'.

In this note, we describe a variant of this approach, which should result in a reduction of the systematic error. In order to be specific, we describe the method as it applies to a neural network approach for separating the classes. The technique can, however, be used with little modification for other methods of separation.

\section{Standard Neural Network Approach}

We now describe in slightly more detail the use of a simulated feed-forward neural network [1] for estimating the fraction of a specific class in a given data sample, and also for estimating the systematic error. In this section, we concentrate on the example from high energy physics of hadronic production in $\mathrm{e}^{+} \mathrm{e}^{-}$reactions, proceeding via $\mathrm{b}$ or other quarks.

Each event of the data sample is characterized by a set of numerical values; these could consist of event shape variables, impact parameters, lepton transverse momenta, etc. The network basically provides an algorithm for calculating an output numerical value from the set of input ones for each event; these input values correspond to the variables mentioned above. The output, which is constrained to be in the range from, say, zero to 1 , is a function not only of the inputs but also of some parameters of the network, known as weights and thresholds.

The network is 'trained' with some known examples of the two categories; in our type of example, these would typically be from Monte Carlo simulations of the two classes of events (b quarks, and others). This training consists of varying the weights and thresholds in such a way as to try to make the output value large (for example, 1) for b quark events, and small (say, zero) for light quarks. More specifically, we may minimize the cost function

$$
C=\sum_{i}\left(z_{i}-w_{i}\right)^{2}
$$

where $z_{i}$ is the network's output for the $i^{\text {th }}$ event and $w_{i}$ is the wanted value ( 0 or 1 above); the summation is over all the events. 
Once the network has been trained, the values of the optimal weights and thresholds are kept fixed, and the network is presented with the real data. Then each event can be tagged as either $b$ or non-b induced, depending on the numerical value of its output. Alternatively, the fraction $f$ of b events in the entire data sample can be estimated, for example, by comparing the observed distribution of the network's output with those expected for samples of pure $b$ and pure non-b events (see Fig. 1). These expected distributions are in general obtained by Monte Carlo simulation.

Any such fitting procedure will yield a statistical error $\sigma_{\mathrm{f}}$ which depends on the number of events in the data sample, the degree to which the Monte Carlo distributions for $b$ and non- $b$ events differ, and possibly also on the numbers of Monte Carlo events.

Now we turn to the systematic errors. It is likely that the Monte Carlo generation for our two samples involves various constants whose values are not exactly known. These could include, for example, the lifetime of beauty particles, features relating to the way $\mathrm{b}$ and light quarks fragment into hadrons, the efficiency for identifying leptons, etc. Then the network is provided with Monte Carlo samples in which each of the relevant constants has been altered by its error ${ }^{1)}$.

For each of these new pairs of samples, the data is used to obtain the fitted fraction $f_{j}$, where $j$ labels which Monte Carlo constant was changed. The contribution to the systematic error due to the uncertainty in this parameter is then $f_{j}-f$ (where $f$ was the previously fitted fraction, obtained with the best values of the Monte Carlo constants), provided the statistical error coming from the finite numbers of Monte Carlo events is negligible; otherwise it is smaller.

Finally all the contributions due to the different systematic effects are generally assumed to be uncorrelated ${ }^{2}$. Then the total systematic error on $f$ is given by

$$
\sigma_{\text {sys }}^{2}=\sum_{j}\left(f_{j}-f\right)^{2}
$$

where the summation is over the various contributions to the systematics.

As an aside, we note that the neural network is simply a mechanism for dividing the different regions of the multi-dimensional space of the input variables, in order to achieve an optimum separation of the different classes of events. The way that the numerical algorithm of the network is defined makes the possible divisions of this space very flexible.

\section{Improved Approach}

That the above approach is not optimal in some circumstances can be seen from the following illustration. Mainly for ease of drawing, we consider an example where the events are each characterized by two input values $\mathrm{x}$ and $\mathrm{y}$. Furthermore, we assume that for the central values of the Monte Carlo constants, the regions of $x-y$ space occupied by the classes A and B (for example, due to the two types of quark categories) are well

1) In practice it may be difficult to know what is a reasonable range for each of these Monte Carlo constants. This is a general problem, however, and is by no means specific to the improved technique we describe below.

2) This assumption is often incorrect. However, in view of the previously mentioned difficulty in estimating even the errors on the Monte Carlo constants, the off-diagonal elements of their error matrix are generally ignored, leading to the 'uncorrelated' approach. Again this problem is not intrinsic to our improved method. 
separated (see Fig. 2a). Then the trained network will divide up the space optimally if it defines everything below the straight line $S$ as a type $A$ event.

Now we introduce the effect of systematics, caused by the uncertainties in our Monte Carlo constants. These will change the A and B distributions; we assume that they are moved in the $\mathrm{x}-\mathrm{y}$ plane as shown in Fig. $2 \mathrm{~b}$ or $\mathrm{c}$.

The problem is that the standard method for systematic errors analyses the data of Fig. $2 \mathrm{~b}$ or $\mathrm{c}$, using the best algorithm defined from Fig. 2a. Thus with the straight line $\mathrm{S}$, we misclassify events at the edges of the $\mathrm{B}$ distribution. This is so even though the two classes of event could be completely distinct, for all reasonable values of the Monte Carlo constant responsible for moving around the distributions as shown in Figs. $2 \mathrm{~b}$ and c. Figure $2 \mathrm{~d}$ shows the composite distributions for classes $\mathrm{A}$ and $\mathrm{B}$, obtained by allowing such a variation of the Monte Carlo constant. Clearly it is possible to train a new network so that it is capable of distinguishing the two classes in Fig. 2d, for which it could divide the $\mathrm{x}-\mathrm{y}$ space as shown by the curve $\mathrm{C}$; the separation is now complete.

It is now clear what the new approach for reducing systematic effects should be: 1) Train the network with samples of events which have been generated using the full range of Monte Carlo constants. That is, for each event, all the relevant Monte Carlo constants are varied from their central values according to their allowed distributions. These could be Gaussians with specified widths, or uniform within given ranges, or whatever. Also it is straightforward to include any known correlations between the Monte Carlo constants. 2) Determine the best value of the fraction $f$ and its statistical error, by using the trained network from 1), with the data sample. For this, the output distribution for the data should be compared with those for classes A and B, which were generated by Monte Carlo with the best values of the Monte Carlo constants (rather than with the varying values as described in 1) above).

3) Determine the systematic error as described in Section 2 above, again using Monte Carlo samples that have been produced with constants which are not allowed to vary during the generation of that sample. Of course the separate samples use different values of these constants (as in Section 2).

The effect of using a network which is trained on the full range of the Monte Carlo constants is that it should deal more efficiently with the possible systematic variations. Thus it should produce a reduced systematic error, perhaps at the expense of a slightly enlarged statistical one. The usefulness of this approach then depends on the relative sizes of the statistical and systematic errors.

We also expect to achieve an improved performance in the following situation. Data is being classified by a network on the basis of several variables simultaneously; we assume that these variables are almost uncorrelated. If the variables were used separately, the various estimates of $f$ could have different ratios of statistical to systematic errors. The conventional approach when using all variables simultaneously is likely to produce an answer equivalent to combining the individual estimates with weights which depend only on their statistical errors. The correct weighting procedure would take into account also the systematic errors, and this is what the improved method should be capable of doing. 


\section{A toy example}

We illustrate the situation described in Fig. 2 with the following toy example. We assume that the classes $\mathrm{A}$ and $\mathrm{B}$ are characterized by distributions $D(x, y)$ in the two input variables $x$ and $y$, which consist of uncorrelated Gaussians:

$$
\left.\begin{array}{ll}
\text { Class A : } & D_{A}(x, y)=G(x, 0,4) G(y, 8,1) \\
\text { Class B : } & D_{B}(x, y)=G(x, 0,4) G(y, 12,1)
\end{array}\right\}
$$

where $G(v, \mu, \sigma)$ is a Gaussian in the variable $v$, of mean $\mu$ and of width $\sigma$. This is the situation illustrated schematically in Fig. 2a.

The above represents the situation for the central values of the Monte Carlo constants. We now suppose that the effect of systematics is to rotate the above distributions about the origin by an unknown angle $\theta$ that is Gaussian distributed with mean zero and width $30^{\circ}$. The situations for $\theta= \pm 30^{\circ}$ are illustrated in Figs. $2 \mathrm{~b}$ and c.

We now test how the two separate approaches perform. In the conventional method (Network I), the training samples are generated according to Eq. (3) (Fig. 2a). The second approach (Network II) uses training samples obtained by generating $x$ and $y$ values according to Eq. (3), but rotating them by an angle randomly chosen from the Gaussian distribution $G\left(\theta, 0,30^{\circ}\right)$ (see Fig. $2 \mathrm{~d}$ ); this is the method for improved systematics.

Because the separation curve is more complicated in the second case, the structure of Network II involved 2 hiddden nodes, as well as two inputs and one output. For the straight line separation of the first method, a network with just two inputs and one output was sufficient.

The results for Network I are shown in the upper part of Table 1. For a sample generated according to Eq. (3), 97\% of the events are correctly classified. The reason this was not $100 \%$ is because there is in fact a finite overlap of the two distributions for classes $\mathrm{A}$ and $\mathrm{B}$ as defined by Eq. (3). However, when we rotate these distributions by either $+30^{\circ}$ or $-30^{\circ}$ to simulate systematic effects, the fraction correct drops to $75 \%$.

The second part of Table I is for Network II. When presented with the data defined by Eq. (3) (i.e. unrotated coordinates, corresponding to the central values of the Monte Carlo constants), it performs less well than the first network, achieving only $86 \%$ success. However, for the samples that have been rotated, Network II not surprisingly achieves much better results than the previous network.

The improved performance achieved by Network II is similarly very apparent when comparing the estimated systematic effects. The Table also shows the estimated fraction of class A, by comparing the network's output distribution for a 'data' sample with those for the Monte Carlo events, for each of the three angles of rotation of the Monte Carlo samples; the generated fraction of $\mathrm{A}$ in the 'data' was $\sim 0.50$. For Network I, the estimate using the unrotated Monte Carlo samples is $0.517 \pm 0.017$, but the systematic shift on rotating by $30^{\circ}$ is an enormous 0.3 . For the improved method, the unrotated sample gives $0.508 \pm 0.023$ (i.e the statistical error is slightly worse), but the possible systematic shifts are only at the level of the statistical error. The improvement is striking.

\section{Conclusion}

The improved method described in this note, which incorporates the allowed range of Monte Carlo constants in the definition of the separation algorithm, can result in a smaller systematic error on the estimated fraction of interest. This new approach could be valuable in situations where the systematic error on the answer dominates the statistical one. 


\section{REFERENCES}

[1] D.E. Rumelhart and J.L. McLelland, 'Parallel distributed processing', MIT Press (1986).

\section{Table I}

\section{Summary of Results}

Networks I and II are used to classify events as type A or B. Network I is trained on samples generated according to Eq. (3), while the samples for Network II have also been given random rotations about the origin. The networks are tested with samples generated according to Eq. (3), with rotations of $-30^{\circ}, 0^{\circ}$ and $+30^{\circ}$ respectively (for the three columns of the Table). The entries in the Table give the percentage of events correctly classified; and the estimated fraction $f$ of type A events in the 'data' sample (the generated fractions were 0.510 and 0.504 for Networks I and II respectively).

\begin{tabular}{|l|ccc|}
\hline \multicolumn{1}{|c|}{ Test samples } & \multicolumn{3}{|c|}{ Rotations } \\
\hline & $-30^{\circ}$ & $0^{\circ}$ & $+30^{\circ}$ \\
\cline { 2 - 4 } Conventional & $76 \%$ & $97 \%$ & $75 \%$ \\
(Network I) & $0.216 \pm 0.051$ & $0.517 \pm 0.017$ & $0.178 \pm 0.056$ \\
\hline Random rotations & $91 \%$ & $86 \%$ & $94 \%$ \\
(Network II) & $0.499 \pm 0.019$ & $0.508 \pm 0.023$ & $0.460 \pm 0.018$ \\
\hline
\end{tabular}




\section{Figure captions}

Fig. 1 Schematic distributions of the output $z$ from the network for (a) events mediated by b quarks; (b) events produced via other quarks; and (c) for the data, which consists of an approximately equal mixture of the two classes.

Fig. 2 Schematic diagrams of distributions of events of classes A and B, in terms of two variables $x$ and $y$.

a) For the central value of the Monte Carlo constant. The line S separates the two classes.

b) For a value of $\theta=-30^{\circ}$ for the rotation, simulating the effect of a different value of the Monte Carlo constant. With the same line of separation as from (a), some of the events of class B are incorrectly assigned as A.

c) As for (b) but for $\theta=+30^{\circ}$.

d) The effect of random rotations. The best separation is now achieved by the curve $C$. 

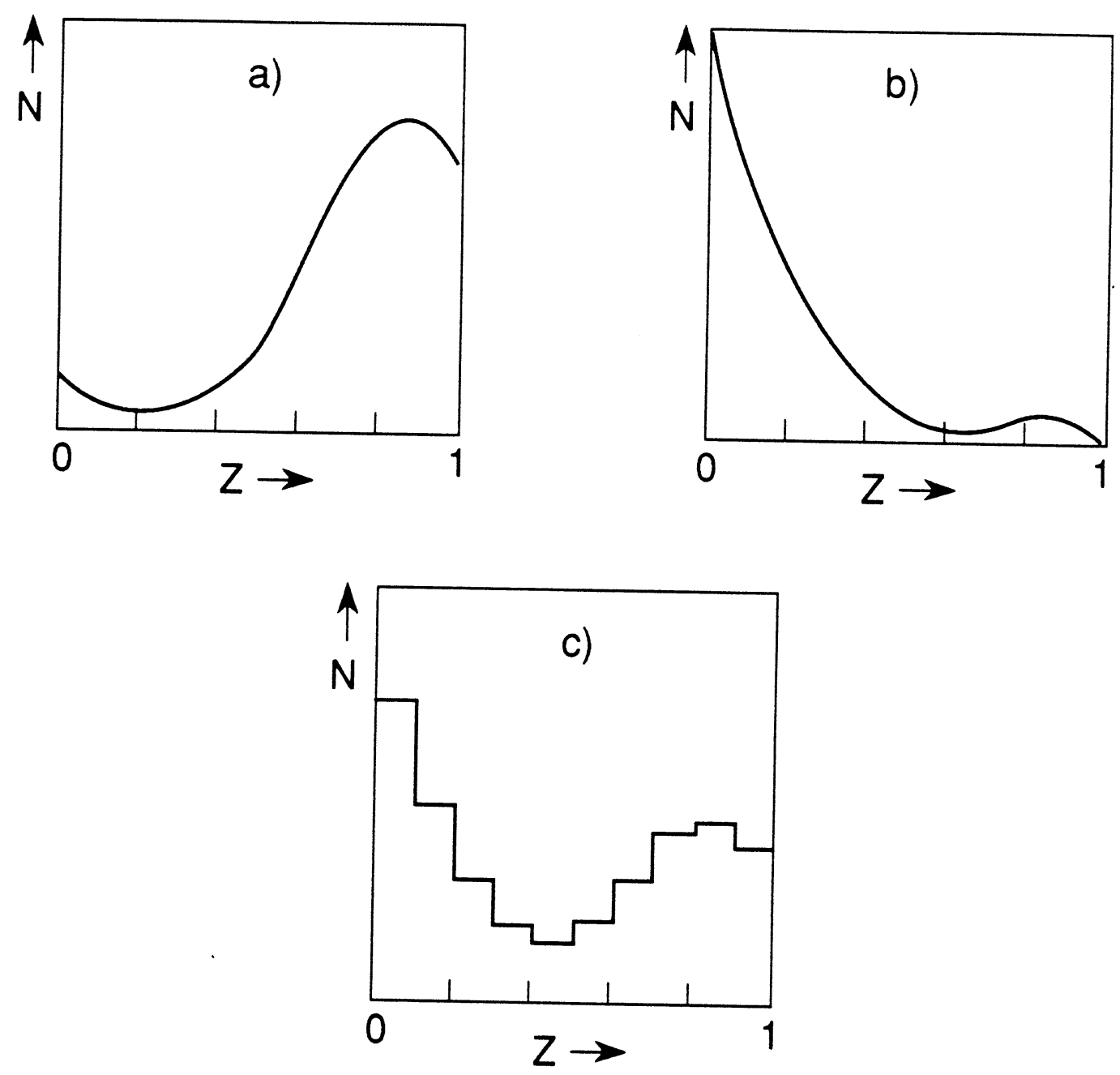

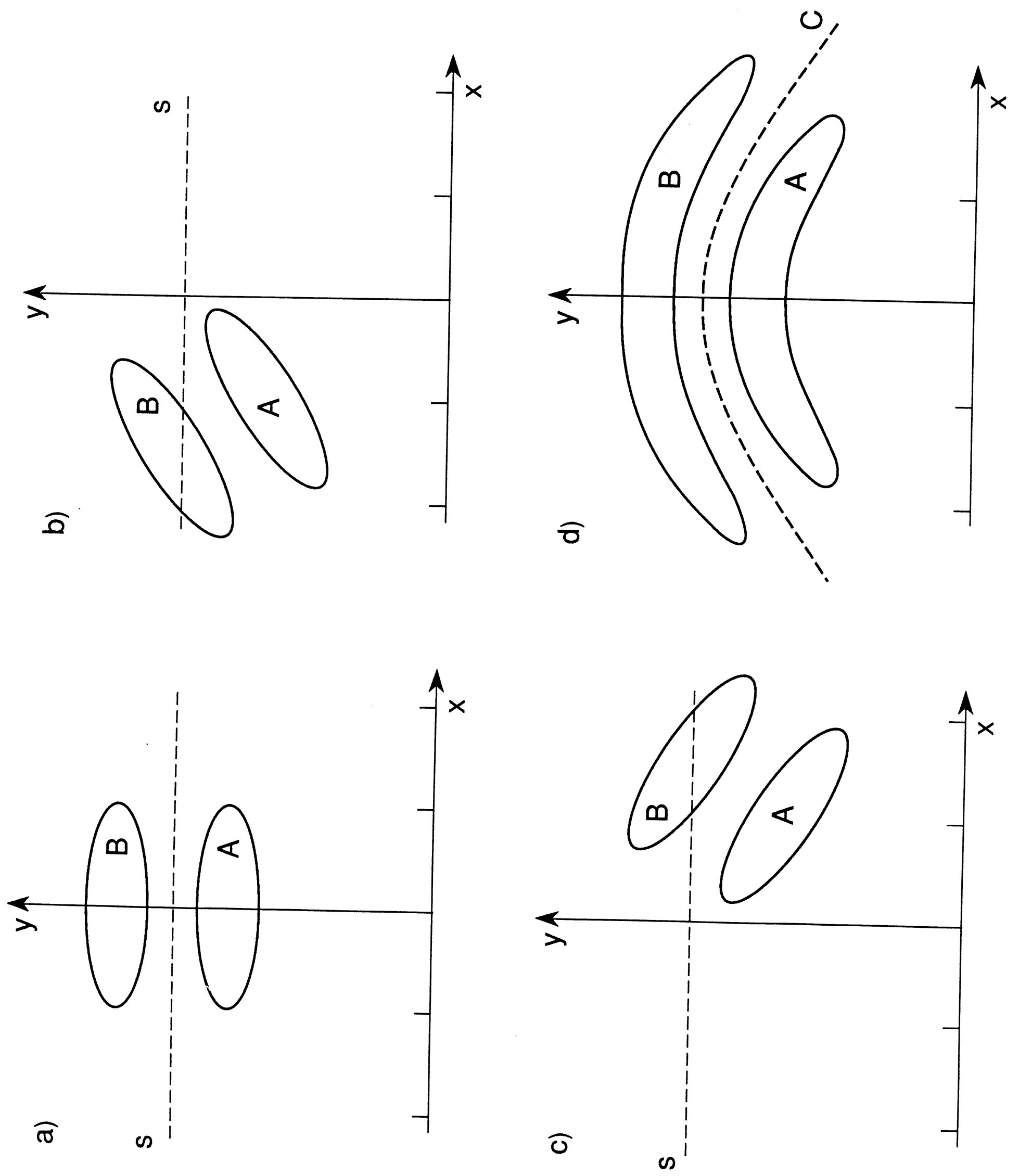